\title{
HUMAN FACTORS AND THE INTERNATIONAL SPACE STATION
}

\author{
Brian Peacock, Sudhakar Rajulu, Jennifer Novak \\ National Space Biomedical Research Institute, \\ Houston, Texas
}

Thomas Rathjen, Mihriban Whitmore, James Maida, Barbara Woolford

National Aeronautics and Space Administration, Johnson Space Center,

Houston, Texas

\begin{abstract}
The purposes of this panel are to inform the human factors community regarding the challenges of designing the International Space Station (ISS) and to stimulate the broader human factors community into participating in the various basic and applied research opportunities associated with the ISS. This panel describes the variety of techniques used to plan and evaluate human factors for living and working in space. The panel members have contributed to many different aspects of the ISS design and operations. Architecture, equipment, and human physical performance requirements for various tasks have all been tailored to the requirements of operating in microgravity.
\end{abstract}

\section{INTRODUCTION}

The NASA space human factors community (SHFE) has the responsibility of contributing to the design and operation of current and future space vehicles. NASA also supports advanced human factors research and development. The tools that NASA uses to identify and prioritize human factors research needs are the Space Human Factors Engineering project plan and the Bioastronautics Critical Path Roadmap. Human factors knowledge is communicated in terms of engineering requirements via the NASA-STD-3000 document.

The project plan and the complementary annual implementation plan address the spectrum of human factors research, development and implementation activities covering physical, cognitive, psychosocial, environmental and systems factors. The human factors component of the critical path roadmap addresses those questions that need to be answered before long duration exploration missions can be undertaken. As in most large organizations human factors personnel in NASA interact with engineering, operations, training and medical functions in a generally seamless way. Similarly, NASA human factors research activity overlaps with a wide variety of disciplines including medicine, psychology and engineering.
The activities of the NASA space human factors engineering function are monitored and guided by a Science and Technology Working Group (STWG) which consists of broad human factors expertise from academia, the military, industry and consulting organizations. These current operations include support for International Space Station and the Shuttle for construction, maintenance and scientific research. In particular the NASA SHFE personnel address acoustics, vision systems, interior volume control, stowage and waste, inventory management, food systems and performance of the multicultural crew. The advanced research and development activities are driven by the challenges of exploration class missions which are characterized by: extended duration in a micro gravity environment, limited communications, minimal or no re-supply and the ever present hostile space environment.

\section{PHYSICAL PERFORMANCE}

The macro spatial design of the ISS is constrained by the challenges of carrying the various modules into orbit in the Shuttle cargo bay. Given these constraints the human factors and habitability specialists at NASA apply the techniques of anthropometry, biomechanics and task analysis to the design of the spatial and 
operational environment. The astronaut population is relatively small in number and the Anthropometry and Biomechanics Facility (ABF) routinely takes a full set of anthropometric and strength measures of all astronaut applicants. Recently these measures have been supplemented by full body scanning of most of the current astronauts. These precise spatial and strength measures are essential for both design and safety. The microgravity environment results in spinal elongation and body shape changes due to fluid shift. These changes are important in the design of Extra Vehicular Activity (EVA) suits as well as shuttle transfer facilities.

\section{Strength Capabilities}

Crewmember mobility and force output capability is limited by EVA clothing and is affected in several ways by the lack of gravity. A combination of lack of ground reaction forces and the need to wear hard shell-type suits changes the capabilities and limitations of humans in space. Hence, strength capabilities of humans are considerably different in space and are unfortunately not comparable or analogous to human strength data from an earth gravity environment. In addition, crewmembers are required to perform certain unique tasks such as satellite retrieval or transport heavy masses in space. There are no analogous tasks in a $1-\mathrm{g}$ environment that can be easily modified to understand how humans would be limited while performing such micro-g specific tasks. Hence, the $\mathrm{ABF}$ has been conducting research to gather data (Rajulu et al., 1994, Rajulu, et al., 1993; Rajulu, 1999) and to develop anthropometric and biomechanical models (Rajulu and Klute, 1992; Gonzalez et al., 2001) for micro- and partial gravity environments. These models can be applied to the prediction of performance capabilities under micro and low gravity conditions and with alternative designs of EVA suits, hardware, and work practices. Effective use is made of micro gravity simulators such as the frictionless surface simulator and the KC 135 airplane. This "vomit comet" performs a series of climbs and dives providing up to 25 seconds of zero $\mathrm{g}$ and $2 \mathrm{~g}$ conditions.

\section{Physical Restraints and Mobility Aids}

The micro gravity environment presents problems of postural stability while crew members perform either high force tasks or precise tasks as with glove boxes. The maintenance of an appropriate posture for extended time periods can lead to fatigue and a decrement in performance capability. Strategically placed restraints and mobility aids, such as foot restraints or hand rails are key to both the maintenance of comfortable postures and for exerting the higher forces needed to manipulate and transfer large mass objects such as racks. The human factors community has been intimately involved with task analysis, equipment and restraint design to facilitate such micro gravity tasks. The involvement of the human factors community was so appreciated by the crew members that they presented Dr Whitmore with the prestigious Snoopy award for her contributions.

A series of ergonomic evaluations of existing Shuttle and potential ISS crew restraints was conducted to identify the human factors requirements for crew restraint design specifically for use with the glove boxes (Whitmore, McKay and Mount, 1995). The experimental work on these studies included video analysis of working posture, human modeling analysis, and a compilation of crew comments and questionnaire responses. The most significant accomplishment of this effort was determining the need for two- (optional three-) point restraining of crew in order to accommodate comfortable crew posture and repetitive, fine motor task control performance in micro gravity. The other significant guidelines were simplicity, stability, flexibility, and easy ingress/egress. A concept with two-point restraining used for the robotic operations onboard the Shuttle was modified and used at the glove box. Currently, this modified two-point crew restraint is being used for all the robotic operations during the ISS assembly missions. In addition, findings and lessons learned from these evaluations have been provided to the developers for two concepts that are being developed one for the ISS robotics operations in the Cupola, a module being developed by one of the International Partners, and one for the Life Sciences Glovebox, a payload module. These ergonomics evaluations not only contributed to the design of 
more effective and safe crew restraints for confined workstations but also helped promote the awareness of ergonomics in optimum glovebox and crew restraint designs. The activity also contributed to the refinement of methods for quantifying on-orbit observational data.

\section{ARCHITECTURE AND HABITABILITY}

The interior of the International Space Station supports activities such as sleeping, eating, personal hygiene, exercising, materials transfer, maintenance, scientific experimentation, stowage and so on. A particularly challenging problem in the space environment that is relevant to all of the tasks performed on ISS is associated with spatial orientation or knowing how one's body is positioned relative to the immediate or extended surroundings. Without the contribution of the usual ground-based vestibular cues, the astronaut is forced to rely exclusively on visual cues. However, this strategy is undermined by the fact that the ISS is designed in accordance with a modularity requirement. In other words standard hardware "units" such as racks, trays, boxes can be traded and exchanged for other locations on ISS. This design requirement was levied on ISS in order to facilitate adaptability of the hardware, extended use over time, and efficient human operations. Unfortunately, the modularity results in uniformity across the visual field and thereby reduces meaningful visual cues. Inadequate spatial orientation can result in human user errors as benign as irritation associated with accidentally navigating down the incorrect pathway only to have to double back. However, this same error can be life threatening if it occurs during an emergency evacuation from ISS. The countermeasures for these challenges of "neuro-vestibular dysfunction" include the provision of global orientation cues in the ISS modules and passageways to provide a continuous awareness of relative and absolute orientation, as well as more specific information indicating distinct emergency escape routes, and finally to training and drills to ensure that navigation during emergencies is well practiced. The global orientation cues are simple and intuitive - parts of the structure are always white for the 'ceiling' or 'overhead' position and the corresponding parts are colored for the 'floor' position. The floor colors are used to code the specific module.

Since a Shuttle and a Soyuz space craft may both be docked to ISS at the same time, and specific crew members are assigned to each vehicle for emergency evacuations, it is important to be able to locate the proper emergency exit. Coding signs to show the way to the exits in three dimensions presented a challenge.

\section{MODELING}

A major challenge for Extra Vehicular Activity (EVA) is the 90 minute night and day cycle. The visual difficulties include visual adaptation and appropriate supplemental lighting. There are also difficulties due to glare, either direct or reflected, shadowing, and the transitional periods between day and night. The human factors modeling group have constructed precise models of the temporal changes in the visual environment thus enabling the detailed planning of activities. When there is deviation from a planned activity, real time modeling can be used to predict exactly what the visual circumstances and difficulties will be and whether or not a task can be completed satisfactorily. Robotic operations are also sensitive to the visual aspects of an environment particularly when indirect viewing with cameras is critical. The modeling is used by mission planners to determine the effectiveness of available lights and cameras to see targets and alignment guides.

Human modeling is also applied during design activity to supplement or replace the costly and less versatile use of crew members in analog devices. Such methods have been used in the evaluation of designs for physical and visual access. A recently convened "Interior Volume Control" function is tasked with the maintenance of adequate operational and emergence access as the various pay loads are installed in the ISS. An associated problem comes with the temporary stowage of equipment, material, tools and trash is the space constrained dynamic environment. Again modeling of the interior environment, with precise human models facilitates important design, location and operational decisions. These same human modeling techniques 
have been applied to EVA activities, both for mission planning as well as for training. A human model can save time and money when appropriately used as a substitute for a real human. (Maida 2001; 1999)

\section{INTERNATIONAL PARTNERS}

The decision to pursue international collaboration in the design and operation of the ISS brings additional challenges to crew members, mission operations, designers and the human factors community. Because of the lack of availability of an emergency return vehicle the operational staff of the Station is limited to 3 crew members, who can use the Soyuz if needed. The makeup of crews during the current construction phase requires delicate attention to selection, training, management and political sensitivities. The international partners include the USA, Russia, Japan, Brazil and the European Space Agency. Each of these partners contributes to the design and construction of the station modules and expects a balanced assignment of crew members. International management styles differ, there are language difficulties, there are even differences in the engineering units needed for design. Representatives of the NASA space human factors community regularly interact with counterparts in other countries to resolve design and operations challenges. Some of the challenges include the selection of exercise equipment, food, tables, communications arrangements and personal living quarters as well as the delegation of routine and contingency duties.

Particular challenges that have many earth bound equivalents are those of crew member productivity, operation quality, health and safety. The activities on the station include, in approximate order of priority, sleep, food, exercise, medical monitoring, personal time, construction, maintenance, scientific experimentation and public affairs, including the role of tour guide. Naturally the scientific community desires efficiency in the conduct of their experiments that are the fundamental purpose of the venture. However, like all the best made plans things don't always go according to plan and the highly motivated crew members sacrifice personal time to "get the job done." As mission duration increases to 6 months or a year, the cumulative effects of microgravity, confinement, isolation and contingency activities will take their toll on crew members. Thus activity scheduling and close monitoring of the multinational crew behavior and performance becomes an operational necessity and a goldmine of human factors knowledge that will be critical in the planning of long duration exploration missions.

\section{REFERENCES}

Gonzalez, L. J., Maida, J., Rajulu, S., (2001) "Predicting Strength and Fatigue For Suited and Unsuited Conditions From Empirical Data", Bioastronautics Investigators' Workshop, January 17-19, 2001, Galveston, Texas, pp. 2223.

Maida, J.C., L. Javier Gonzalez, S.L. Rajulu, and E. Miles (2001), Predicting Fatigue for Isolated Joints Wearing an Extra-Vehicular Mobility Unit (EMU)., SAE Digital Human Modeling for Design Engineering Conference, Arlington, VA.

Maida, J., Novak, J., Mueller, K., (1999). "Effects of Training Using Illumination In Virtual Environments", Human Computer Interaction, HCI 1999 Proceedings.

Maida, J., (1999) “An Illumination Modeling System for Human Factors Analyses", Electronic Proceedings of the 1999 International Conference on Computer-Aided Ergonomics and Safety, Barcelona, Spain, May 19-21, 1999.

Morgan, D., Wilmington, R., Pandya, A., Maida, J. and Demel, K., (1996) "Comparison of Extravehicular Mobility Unit (EMU) Suited and Unsuited Isolated Joint Strength Measurements", NASA Technical Paper 3613, June 1996.

Novak, J. B., Balmer, D., Morphew, E., Smart, K. (2001) "Operational Habitability For Long Duration Space Flight". Presented at the Aerospace Medical Association $72^{\text {nd }}$ Annual Scientific Meeting, Reno, Nevada, May 2001

Novak, J.B. (2000) Human Engineering and Habitability: The Critical Challenges for the International Space Station. Aviation, Space and Environmental Medicine. V.71(9), Section II, p.A117-A121.

Novak, J.B. (2000) Summary of Current Issues regarding Space Flight Habitability. Aviation, Space and Environmental Medicine. V.71(9), Section II, p.A131A132..

Novak, J. B., Liddell, G. \& Sampaio, C. (1997). Human Engineering Applied to International Space Station 
Design. 27th International Conference on Environmental Systems, Lake Tahoe, Nevada. \{SAE Technical Paper Series No. 972400 \}

Rajulu, S.L., Klute, G.K., and Fletcher, L. (1994), "Evaluation of COSTAR Mass Handling Characteristics in a Frictionless Environment - A Simulation of the Hubble Space Telescope Repair Mission," NASA Technical Paper 3489, NASA-JSC

Rajulu, S.L, Poliner, J., and Klute, G.K. (1993), "Loads Produced by a Suited Subject Performing Tool Tasks Without the Use of Foot Restraints," NASA Technical Paper 3424, NASA-JSC.

Rajulu, S.L. (1999), "Light-Weight Seat Lever Operation Characteristics", NASA Technical Paper 209577, NASAJSC

Rajulu, S.L., and Klute, G.K., (1992), "Biomechanical Analysis of Locomotion Patterns in Partial Gravity Environments," Proceedings of the Human Factors Society 36th Annual Meeting, Atlanta, GA.

Smart K., Novak, J. B., Carr, D., Bowen, C. (2001) ISS Emergency Egress - A System for Crewmember Pathway Indication, 31st International Conference on

Environmental Systems, Orlando, Florida \{SAE Technical Paper Series No. 2001-01-2184\}

Whitmore, M., McKay, T.D. and Mount, F. E. (1995).

Ergonomic evaluation of a Spacelab glovebox.

International Journal of Industrial Ergonomics, 16, 155-

164. 